\title{
Study of Altered Mechanical Properties of Articular Cartilage in Relation to the Collagen Network.
}

\author{
$\mathrm{JP} \mathrm{Wu}^{1, \mathrm{a}}$ \& TB Kirk ${ }^{2, \mathrm{~b}}$ \\ 1,2 School of Mechanical Engineering, the University of Western Australia, Perth, Australia. \\ awping@mech.uwa.edu.au, birk@mech.uwa.edu.au
}

Keywords: articular cartilage, biomechanics, 3D collagen network, fibre optic confocal microscopy.

\begin{abstract}
Articular cartilage is a semitransparent elastic material that covers on the two articulating bones in synovial joints. It acts as a cushion between the bones that transfers loads from one to another while attenuating dynamic stresses and providing almost frictionless contact surfaces for normal use of synovial joints without pains. Osteoarthritis causes a chronic joint pain and it is mainly due to malfunction of articular cartilage. The mechanical function of articular cartilage is derived from its unique microstructure. Therefore, study of the relationship between the mechanical function and microstructure of articular cartilage comprehends the aetiology and pathology of osteoarthritis. Confocal microscopy permits studying the internal microstructure of buck biological tissues without tissue sectioning and dehydration. This provides a way to study the relationship between the mechanical function and microstructure of articular cartilage. Using a fibre optic laser scanning confocal microscope, this study examines the pathological status of articular cartilage in relation to the mechanical function and 3D collagen network of articular cartilage. The results show that the 3D collagen structure and the mechanical function are different between normal and arthritic cartilage. Loss of the integrity of the 3D collagen network is closely related to cartilage softening.
\end{abstract}

\section{Introduction}

Osteoarthritis (OA) is synonymous of the malfunction of articular cartilage (AC). At an early stage, it is typically shown as mat of articular surface and softening of AC, which are the result of degeneration of the microstructure of $\mathrm{AC}$ [1]. At a late stage, $\mathrm{OA}$ is often characterized with worn off the AC [2], which leads to a bone against bone operation and sever pains in synovial joints. Today, an effective treatment of $\mathrm{OA}$ is dragged by a lack of a tool in clinic to assess the microstructural and functional degeneration of AC involved in early OA.

A synovial joint is a mechanical system. AC acts like a cushion between the two articulating bones. It transmits loads from one to another, attenuate dynamic peak stresses and provide almost frictionless surfaces for normal use of synovial joint without pains [3]. The mechanical function of $\mathrm{AC}$ has a central role in the normal use of synovial joints, and it is derived from the integrity of the tissue's microstructure and composition [4]. AC mainly contains chondrocytes, proteoglycans (PGs), collagen fibres (Col), and up to $80 \%$ of water [5]. Chondrocytes manufactures and regulates $\mathrm{AC}$ to meet its physiological and mechanical requirements. PGs are highly negative charged macromolecules covalent with the water molecules and generate a swelling pressure in AC. The swelling pressure is reinforced by the $3 \mathrm{D}$ collagenous network that anchors to the subchondral bone. Therefore, AC is provided with the resilience and loading capacities.

Creep behaviour is a unique mechanical property of $\mathrm{AC}$ and often used to study the mechanical function of the tissue. When a constant load is applied to AC, AC responses an instantaneous deformation, followed by a time-dependent creep deformation until the tissue stress reaches equilibrium. Intrinsically, the instantaneous deformation is resulted from the instant outflow of the proteoglycan gel due to the hydrostatic gradient caused by the applied load in the AC [4]. The following time-dependent creep deformation is derived from the delaying of the outflow of the proteoglycans gel due to the dragging and friction of the 3D collagen matrix to the proteoglycan gel. With time, the volume of the 3D collagen network gradually becomes smaller and smaller 
while the resistance to the outflow of the proteoglycan gel gets larger and larger. The creep stops when the hydrostatic gradient diminishes, and the stresses developed in AC can balance the applied load. At this time, there is no fluid outflow inside the AC. It is called the stress equilibrium.

Adult AC does not contain blood vessels, nerves and lymph [6]. The instantaneous deformation behaviour of AC instantly increases the contact area of the two articulating bones during joint loading. This reduces the contact stresses, and absorbs dynamic peak forces in the bones. The creep deformation followed permits the loads to be transferred gradually and smoothly from one segment to another. At tissue stress equilibrium, normal AC maintains a certain deformation thickness. Therefore, the subchondral bones underneath $\mathrm{AC}$, which contains blood vessels and nerves, cannot be disturbed to cause joint pain. Collagen possesses great tensile strength. It forms the scaffold of AC. The orientation of the collagen fibres in the superficial zone is critical to the wear and shearing resistance of $\mathrm{AC}$ [7]. Elsewhere, the integrity of the collagen scaffold is central to the loading capacity of $\mathrm{AC}$ [3]. Using a 3D imaging techniques developed [8], this study investigates the creep property of $\mathrm{AC}$ in relation to the collagen network and physiological status of $\mathrm{AC}$, and it aims to comprehend the aetiology of AC and develop a diagnostic tool for early OA.

\section{Methods}

Fibre optic laser scanning confocal microscopy (FOCM, Optiscan Pty Ltd, Melbourne, Australia) was modified with a specifically designed test apparatus attached to the stage platform, as shown in Fig.1. A constant load was applied to an AC specimen using a pneumatic actuator (PA) with a digital pressure regulator (Festo Pty Ltd, Australia). A load cell (Entran Sensors and Electrons, Fairfield, USA) connected to the third channel of a dynamic signal analyzer (DSA) (Agilent Technologies, CA, USA) was placed underneath the specimen to monitor and ensure the load on the cartilage to be constant. The deformation response of AC was recorded by the second channel of the DSA via a linear variable differential transducer (LVDT) (RS Components Pty Ltd, NSW, Australia). The first channel of the DSA records the time interval.

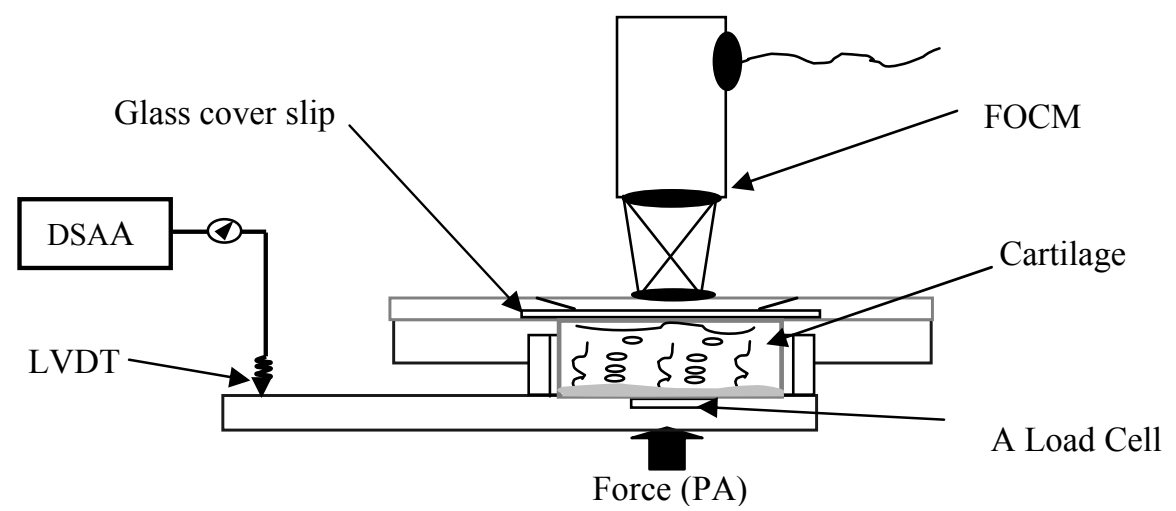

Fig 1. Modified fibre optic laser confocal microscope was used to study in situ microstructural integrity of the $3 \mathrm{D}$ collagen network in relation to the physiological and mechanical properties of articular cartilage.

The cartilage specimens were prepared as approximately $\varnothing 3 \times 4 \mathrm{~mm}^{3}$ cylinders attached to the subchondral bones. Eighteen normal cartilage specimens (ICRS grade 0, Fig 4N) were obtained from the central weight bearing regions of ten femoral heads of two-year-old cows within 24 hours of slaughter. Fourteen arthritic specimens were harvested from the regions of twelve femoral heads (removed from joint replacement surgery) that showed slight mat (ICRS grade 1, Fig 4A).

All the specimens were preserved in 10\% buffered formalin solution (BFS) for 24 hours before immersed into $0.2 \%$ Phosphomolybdic for another 24 hours to reduce the brightness interference of the chondrocytes for imaging the collagen fibres [8]. After washed by $9 \%$ saline water, the specimens were placed into a specially designed container, which maintains the hydration of the specimens all time, to acquire two sets of confocal image stacks at pre-loading and loading status of 
AC. The image acquisition was made by use of a 60x/NA 0.9 (Olympus, Japan) water immersion lens and a laser of $488 \mathrm{~nm}$ and $514 \mathrm{~nm}$ via a reflectance channel. The imaging step size was $0.541 \mathrm{um}$. Using the 3D imaging technique developed previously [8], two sets of the 3D collagen network corresponding to the preloading and loading status were reconstructed (Figs $2 \mathrm{~N}$ and $3 \mathrm{~A}$ ). A load of 3 and 4 Newton was applied respectively to the arthritic and normal cartilage. All the images at loading status were acquired at the time when tissue stress reached equilibrium. Using Optimas (Media Cybernetics Inc, MD, USA), the deformation strain of the collagen network was measured. After this, traditional histology using Alcian Blue stain was further carried out to grade the physiological status of AC in International Cartilage Repair System (ICRS), as shown in Fig 4.

\section{Results}

The 3D collagen network has a close relationship with the function and physiology of AC. Normal AC (ICRS grade 0, Fig 4N) contains an undisrupted collagen matrix, shown in Fig 2. This grants a greater loading strength to the AC, as shown in Fig 5. The integrity of the collagen network in normal AC has also allowed the collagen network to withstand a 4 Newton mechanical load without structural disruption, as shown in Figs $2 \mathrm{NL}$ and 2NLxy. At the tissue stress equilibrium, the deformation strain of the collagen network in normal AC was about $-46 \% \pm 8.4 \%$.
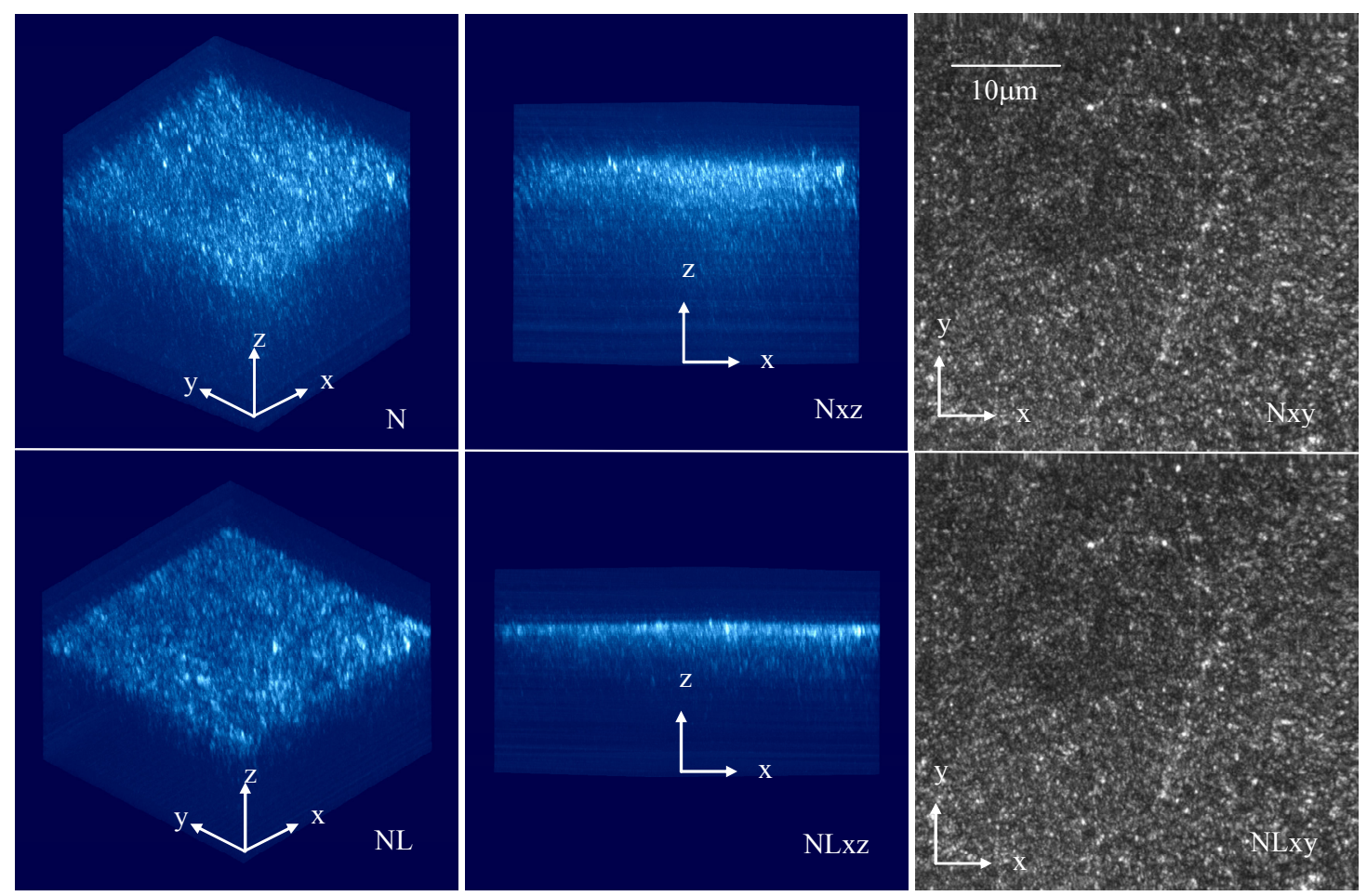

Fig 2. The collagen network in normal AC ICRS grade 0 possesses structural integrity, shown in image $3 \mathrm{~N}$. The integrity of the collagen network has allowed it to withstand a four-Newton-load without disruption, shown in images NL and NLxy. The collagen network deforms when it was under load, shown in images Nxz and NLxz.

Loss of integrity of the collagen structure was clearly seen in early arthritic cartilage (ICRS grade 1), as shown in Figs 3A and Axy. It has led to the disruption of the articular surface in the cartilage (Fig 4A) and a greater deformation of the collagen network (Figs 3Axz and ALxz) at a 3 Newton mechanical load. At stress equilibrium, the deformation strain of the collagen network in arthritic cartilage was about $-68.4 \% \pm 9.1 \%$, which is about $48 \%$ larger than that of the normal cartilage. Disruption of the collagen network in the arthritic cartilage at ICRS grade 1 has also resulted in a seriously structural destruction to the collagen network at a 3 Newton load, as shown in Figs 3AL to ALxy. 

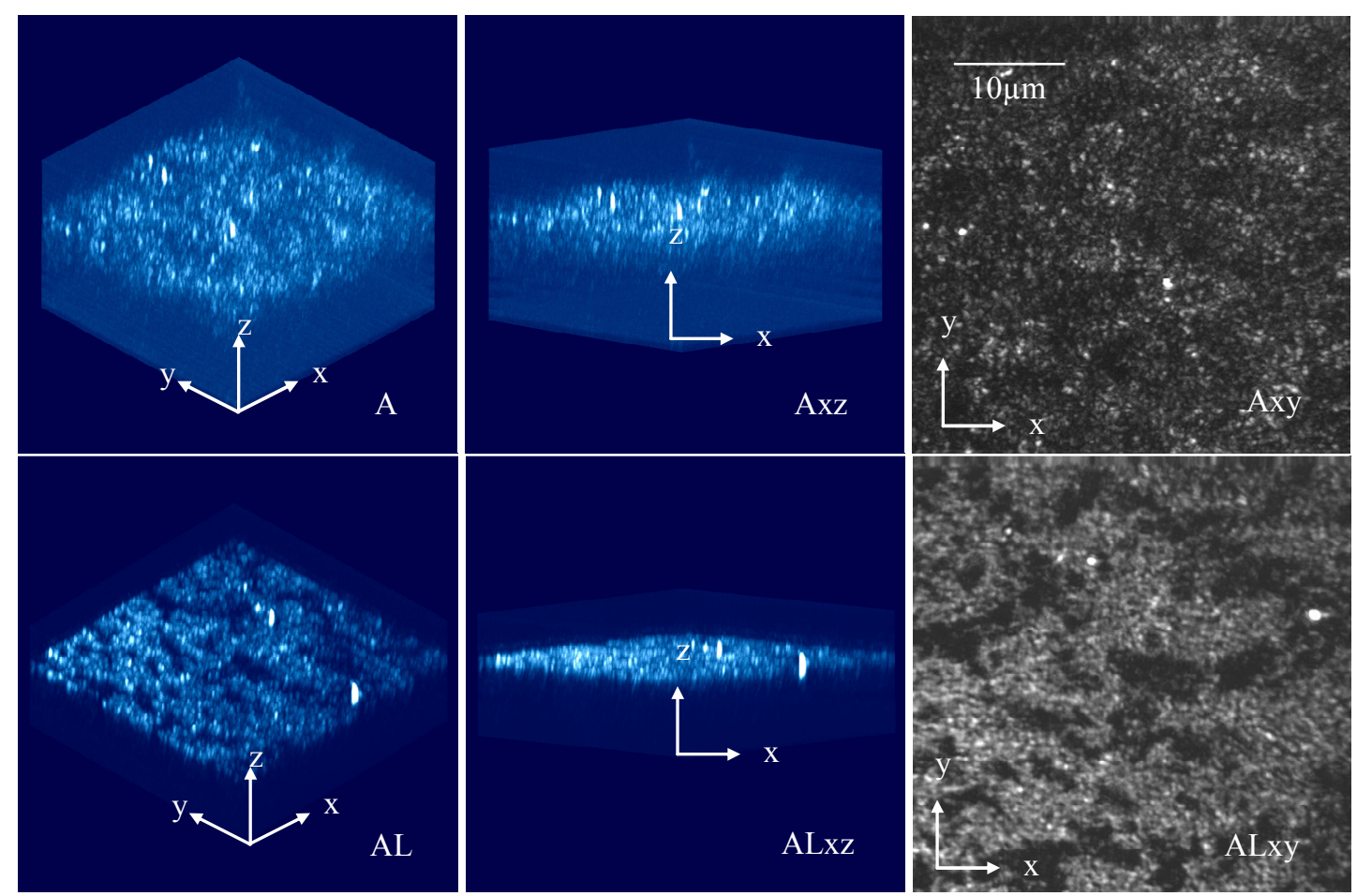

Fig 3. Disruption of the collagen structure was observed in the arthritic cartilage at ICRS grade 1, as shown in image 4A. Loss of the integrity of the collagen network has become more obviously when a $3 \mathrm{~N}$ load was applied to the AC, as shown in images 4AL and ALxy. The deformation strain of the arthritic collagen network is much bigger than that of normal cartilage, shown in images 4Axz and ALxz.
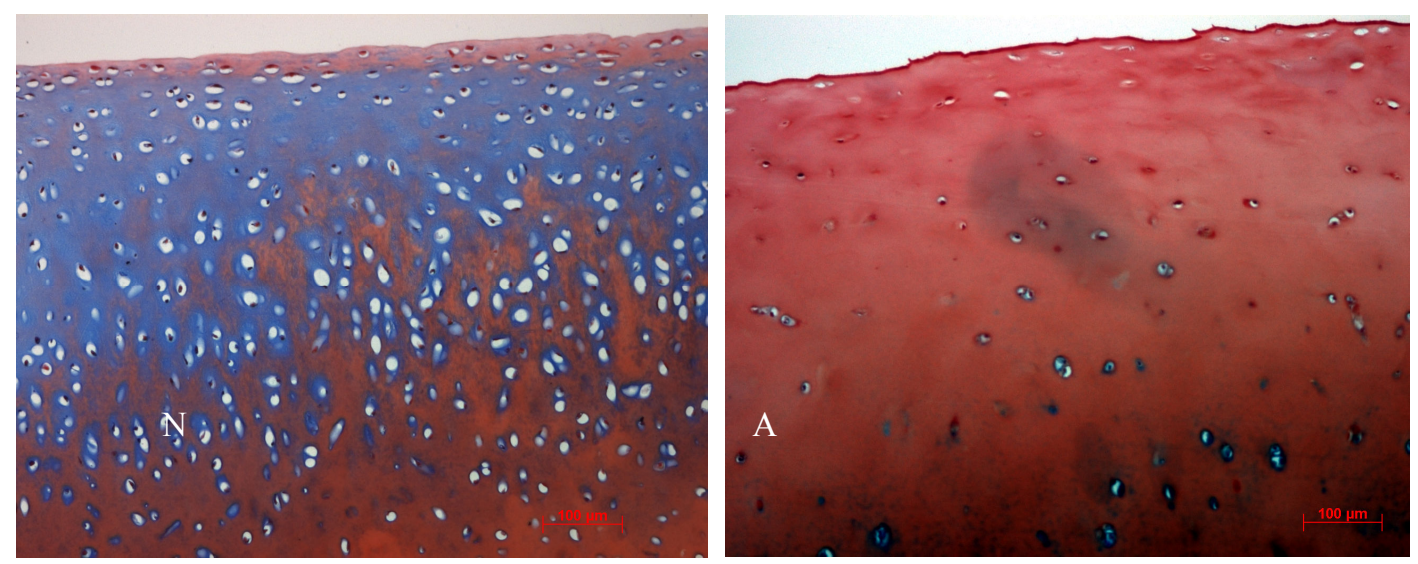

Fig 4. Traditional histology used to score the articular cartilage specimens in International Cartilage Repair System (ICRS). N: Normal cartilage, ICRS Grade 0. A: Arthritic cartilage, ICRS Grade 1.

Both the normal cartilage (ICRS grade 0) and arthritic cartilage at ICRS grade 1 demonstrated typical creep behaviours under a constant load, as shown in Fig 5. However, the loss of the integrity of the collagen network in the arthritic cartilage has led to a sharp increase in the initial instantaneous deformation in the cartilage. The subsequent creep strain of the arthritic cartilage is also greater than that of normal AC. At the stress equilibrium, the deformation strain of normal cartilage is about $19.7 \%$ smaller than that of arthritic cartilage at ICRS grade 1 . Further more, the deformation strain of the collagen network in both physiological status, shown in Figs $2 \mathrm{Nxz}$ and $3 \mathrm{Axz}$, is larger than their corresponding physical deformation strain, shown in Fig 5. 


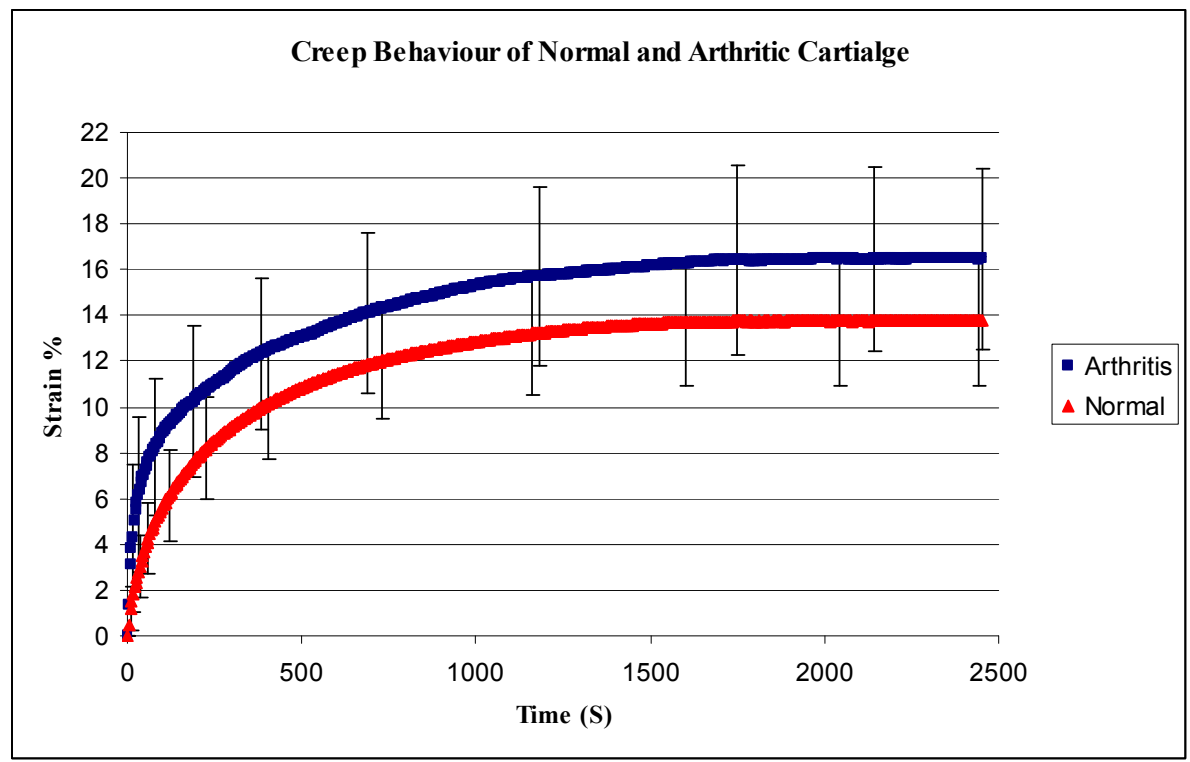

Fig 5. Creep behaviour test show that arthritic cartilage at ICRS grade 1 has a sharp instantaneous deformation strain than that of normal articular cartilage (ICRS grade 0). Its creep deformation strain is also larger than that of normal AC. At tissue stress equilibrium, the deformation strain of the arthritic cartilage is bigger than that of normal AC.

\section{Summary}

The unique microstructural arrangement of AC provides the tissue with exceptional mechanical properties for normal activities. Collagen and proteoglycans are the primary components of the extracellular matrix of $\mathrm{AC}$ where the mechanical loads are exerted on during joint loading. Unlike the proteoglycans, the collagen have a fairly constant concentration during degeneration of AC but its structure and orientation change significantly since the beginning of OA degradation [9]. Therefore, study of the structural integrity of the collagen network under loads is fundamental to comprehend the aetiology of $\mathrm{AC}$ and development of diagnostic techniques for early OA.

Collagen has a similar refractive index to the proteoglycans while they are closely integrated to construct the scaffold of AC [10]. Thus, collagen can not be resolved by traditional optical microscopy. Electro microscopy has a superior imaging resolution for studying the collagen structure of $\mathrm{AC}$ but it requires tissue dehydrating and sectioning. Therefore, it can not be used to study the in situ microstructural integrity of the 3D collagen matrix of AC under mechanical loading. Confocal microscopy offers an improved imaging resolution than that of traditional optical microscopy [11]. It also permits studying the internal microstructure of $\mathrm{AC}$ while the tissue is not scarified physically sectioning and dehydrating. It has permitted for study of the morphological changes of chondrocytes under mechanical compression of $\mathrm{AC}$ [12]. Using a 3D imaging technique developed for studying the 3D collagen network in AC [8], this study examined the in situ integrity of the collagen network in relation to the physiological and mechanical properties of AC. The findings strongly support that disruption of the collagen structure plays an important role in alteration of the mechanical function and physiological states of AC [13]. The study has increased understanding the inter-dependency of the collagen structure and mechanical integrity of AC.

When $\mathrm{AC}$ is under a constant load, the integrity of the $3 \mathrm{D}$ collagen network, as observed in normal AC, controls properly the outflow rate of the proteoglycan gel. Therefore, the AC undergoes a controlled physical deformation, which prevents disturbing the subchondral bone underneath and a joint pain. Conversely, disruption of the collagen network, as found in arthritic cartilage at ICRS grade 1, allows the proteoglycan gel to expand its volume and causes cartilage softening. During joint loading, the disrupted collagen network can not control the permeability of the proteoglycan gel within the collagen matrix. This leads to a larger physical deformation than normal in the AC. 
Consequently, the subchondral bone containing never and blood system is disturbed so that a joint pain occurs. In this study, the deformation strain of the collagen network has been found to be larger than the physical deformation strain of AC. This proves that proteoglycan gel also participates in forming the loading strength of $\mathrm{AC}$ [13].

In this study, the loading magnitudes $(3 \mathrm{~N}$ loads $\approx 0.43 \mathrm{MPa}$ compression, $4 \mathrm{~N}$ loads $\approx 0.57 \mathrm{MPa}$ compression) were selected to allow imaging the same region of the collagen matrix at preloading and loading status but not the relevant physiological loading magnitudes. The physiological compression of 1.5 to $3.5 \mathrm{MPa}$ measured in human knees [14] is lager than those used in this study. It caused a rapid instantaneous deformation in $\mathrm{AC}$, which migrated the portion of the collagen matrix under examining away from the field of view, and made the study impossible. Also, the use of cow cartilage as controlled normal cartilage is due to the unavailability of normal human cartilage. The joints from different mammalian species have been reported to have very similar function and structure [2]. Therefore, this will not cause significantly effects on the study. However, future study on this topic will look for examining same species cartilage and the possible variation in the results caused due to use of difference species cartilage. Moreover, the FOCM used in this study has allowed development of a confocal arthroscope to act as optical histology for assessing in vivo cellular structure of AC [15]. Therefore, the current study has potential to develop a tool for in vivo assessing the structural and functional integrity of $\mathrm{AC}$ for diagnosis of early $\mathrm{OA}$.

\section{References}

[1] N.D. Broom, M.H. Chen, and A. Hardy: Journal of Anatomy, 199 (2001), p. 683.

[2] T.B. Kirk, A.S. Wilson, and G.W. Stachowiak: Journal of Orthopaedic Rheumatology, 6(1993), p. 21.

[3] G.E. Kempson, in: Adult Articular Cartilage, editor by M.A.R. Freeman, Pitman Medical: London (1979), p. 333.

[4] V.C. Mow and W.C. Hayes: Basic Orthopaedic Biomechanics. New York: Raven Press (1991).

[5] G. Meachim and R.A. Stockwell, in: Adult Articular Cartilage, edited by M.A.R. Freeman, Pitman Medical: London (1979), p. 1.

[6] F.N. Ghadially: Fine structure of synovial joints, London: Butterworth \& Co (1983).

[7] S. Swanson: in: Adult Articular Cartilage, edited by M.A.R. Freeman, Pitman Medical Publishing Ltd: London (1979), p. 415.

[8] J.P. Wu, T.B. Kirk and M.H. Zheng: Journal of Musculoskeletal Research, 8 (2004), p. 167.

[9] J. Bland and S. Cooper: Semin Arthritis Rheum, 14 (1984): p. 106.

[10] A.K. Jeffery, et al: The Journal of Bone and Joint Surgery [Br], 73(1991): p. 795.

[11] C.J.R. Sheppard, and D. Shotton, Confocal Laser Scanning Microscopy, edited by C.J.R Sheppard. BIOS Scientific Publishers Limited, London (1997).

[12] Guilak, F., et al., The deformation behavior and mechanical properties of chondrocytes in articular cartilage. Osteoarthritis and Cartilage, 1999. 7(1): p. 59-70.

[13] N.D. Broom and H. Silyn Roberts: Arthritis Rheum, 33 (1990): p. 1512.

[14] B. Weightman and G.E. Kempson, in: Adult Articular cartilage, edited by M. Freeman, 1979, Pitman Medical Publishing Ltd: London (1979), p. 291.

[15] Jones, C.W., et al: Journal of Musculoskeletal Research, 8 (2004): p. 75. 